\title{
Encephalopathy associated with COVID-19 in a patient with multiple sclerosis
}

\author{
Abdorreza Naser Moghadasi ${ }^{1}$ (D)
}

Received: 12 August 2020 / Revised: 24 September 2020 / Accepted: 8 October 2020 / Published online: 28 October 2020

(c) Journal of NeuroVirology, Inc. 2020

\begin{abstract}
From the beginning of COVID-19 pandemics, the involvement of patient's nervous system with this virus is increasingly reporting. Although various reports are published on affliction of multiple sclerosis (MS) patients with SARS-CoV-2, no report has been published on brain involvement by this virus in MS patients so far. Herein, a 34-year-old patient with MS who experienced the decreased level of consciousness and encephalopathy following COVID-19 involvement has been reported.
\end{abstract}

Keywords COVID-19 $\cdot$ Multiple sclerosis $\cdot$ Encephalopathy $\cdot$ SARS-CoV-2 $\cdot$ IVIG

From the beginning of COVID-19 pandemics in December 2019 , the involvement of patient's nervous system with this virus is increasingly reporting (Ahmad and Rathore 2020). Ye et al. reported a man who hospitalized with a diagnosis of COVID-19 developed loss of consciousness. The brain computed tomography (CT) scan was normal, but in the cerebrospinal fluid (CSF), white blood cells were observed at $0.001 \times 10^{9} / \mathrm{L}$ by $0.27 \mathrm{~g} / \mathrm{L}$ protein and $3.14 \mathrm{mmol} / \mathrm{L}$ sugar. Although the result of the COVID-19 test in CSF was negative, the patient was treated with the diagnosis of COVID-19 encephalitis. Then, he was discharged in good general condition after further examinations and the rejection of other causes (Ye et al. 2020). Also, Rayan El-Zein et al. reported a 40-year-old man without any risk factors and previous illness admitted with a diagnosis of COVID-19 pneumonia and discharged after the treatment. However, he was brought to the hospital 1 day after discharge with a decrease of consciousness level. Magnetic resonance imaging (MRI) and CT scans of brain were normal, but in the CSF, white blood cells were detected with a lymphocyte preference of $85 \%$. Although the polymerase chain reaction (PCR) test of SARS-CoV-2 was negative in CSF, the patient was treated with a diagnosis of the encephalitis caused by COVID-19 due to the exclusion of other causes. Intravenous immunoglobulin (IVIG) was started for the patient. The patient's

Abdorreza Naser Moghadasi

abdorrezamoghadasi@gmail.com

1 Multiple Sclerosis Research Center, Neuroscience Institute, Tehran University of Medical Sciences, Tehran, Iran condition gradually improved. Eventually, the patient was discharged in a good general condition (El-Zein et al. 2020).

Misayo Hayashi et al. also reported a 75-year-old man with a history of Alzheimer's disease who referred to the hospital with a complaint of tremor in his left hand and inability to walk and diarrhea in the same time. Also, the CT scan of lungs showed the evidence in favor of COVID19, confirmed by PCR testing. MRI showed a unique lesion in the splenium of corpus callosum. Unfortunately, he died during the hospitalization due to the pulmonary involvement, despite improving his neurological condition. Due to improving the neurological conditions, the patient was diagnosed with mild encephalitis/encephalopathy with reversible splenial lesion (MERS) caused by COVID-19 (Hayashi et al. 2020).

In addition to patients with encephalitis, the cases with encephalopathy are reported due to causes other than the direct invasion of SARS-CoV-2. This encephalopathy can occur due to high inflammatory activity in COVID-19 disease or hypoxia due to COVID-19-related lung involvement and pneumonia (Berger 2020).

Although the cases with encephalopathy are increasingly reported, no case of encephalopathy associated with COVID-19 in patients with multiple sclerosis (MS) has been reported.

Herein, we reported a patient with MS who experienced the decreased level of consciousness and encephalopathy following COVID-19 involvement.

The patient was a 34-year-old woman with the established MS diagnosis for 15 years. Her disease had been 
initiated with lower limb paresthesia, and at the beginning, Avonex was prescribed for her. Subsequently, due to the frequent relapses of the disease, Betaferon, and then dimethyl fumarate were administered, and finally treatment with rituximab was performed. However, despite all these medication changes, her disease has progressed. The brain MRI revealed multiple confluent periventricular lesions with gadolinium enhancement (Fig. 1a, b, c). Lumbar puncture (LP) was performed. CSF analysis was normal. Oligoclonal bands (OCBs) were present in CSF. Rituximab was discontinued, and the patient was treated with cyclophosphamide $1 \mathrm{~g}$ per month along with $30 \mathrm{mg}$ oral prednisolone per day with a diagnosis of active progressive MS. Before developing COVID-19, the patient
Fig. 1 a, b Brain MRI revealed multiple confluent lesions with gadolinium enhancements. c Cervical and brain stem involvements were seen in cervical MRI. d Chest CT scan revealed lung consolidation. e Numerous old lesions were observed in the new brain MRI
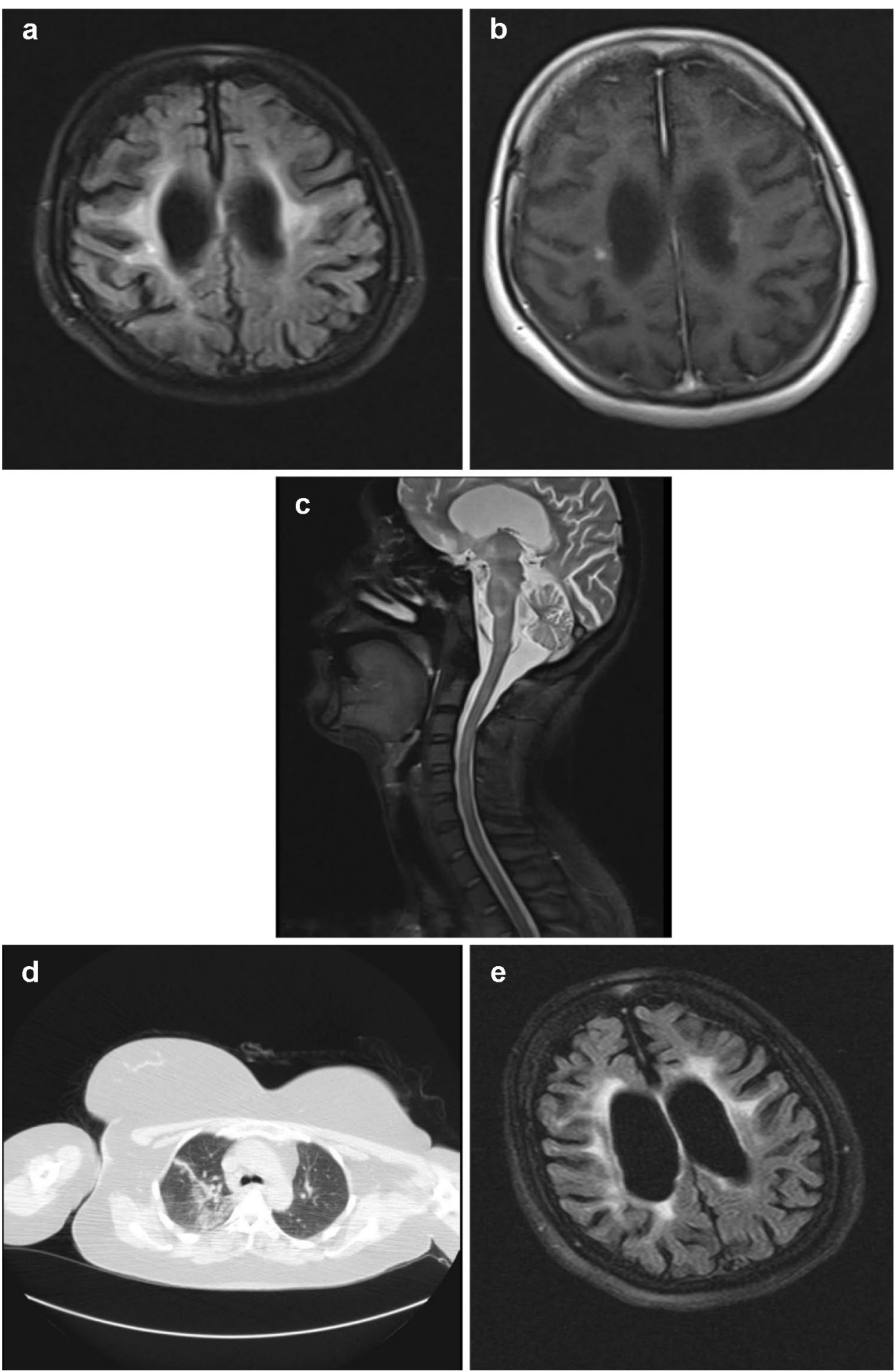
only complained about the recurrent urinary tract infections which improved with oral antibiotics.

The patient totally received 10 cycles of cyclophosphamide. By passing 25 days from receiving the last dose of injective cyclophosphamide, she complained of fever, coughs, dyspnea, generalized weakness, and nausea. In the first examination, the followings were measured: respiratory rate: 20 , pulse rate: 100 , temperature: $38{ }^{\circ} \mathrm{C}$, and $\mathrm{BP}$ : $100 / 60$. Moreover, $\mathrm{O}_{2}$ saturation was $95 \%$ with no oxygen use. Neurologically, the patient had complete time, place, and person orientation. In addition, she had imbalance during walk, and her reflexes had also increased. The muscle power of limbs was four-fifth, which had no difference with the examination performed before COVID-19. The chest CT scan showed bilateral lung involvement especially on the right side (Fig. 1d). So, the patient was hospitalized with COVID-19 diagnosis. In the tests performed, white blood cell (WBC) count was 3100 , lymphocyte $20.9 \%$, C-reactive protein (CRP) $62 \mathrm{mg} / \mathrm{dl}$, and lactate dehydrogenase (LDH) was 737. Notably, nasopharyngeal and oropharyngeal swab real-time polymerase chain reaction (rt-PCR) test of coronavirus was positive. The patient received Azithromycin and Hydroxychloroquine. On day 5 of hospitalization, the patient's fever increased up to $40{ }^{\circ} \mathrm{C}$, and $\mathrm{O}_{2}$ saturation was 93\%. The patient found diminished consciousness level. Afterward, she was localized in response to painful stimulus, and she also had neck stiffness. Routine tests including blood sugar and biochemistry were normal. Subsequently, ceftriaxone and vancomycin were administered for her, and then emergency MRI was performed. In MRI, numerous old lesions were observed in the periventricular area induced by MS, which had no enhancement in the injected view (Fig. 1e). Moreover, LP was performed for her. The CSF pressure was $10 \mathrm{~cm} \mathrm{H}_{2} \mathrm{O}$. In CSF analysis, glucose was measured as 45 , protein as 112 , and $\mathrm{WBC}$ as 0 . CSF smear was negative in terms of bacteria. IgG levels were low in the requested tests. Due to the history of receiving rituximab and low IgG levels, the patient was treated with the IVIG $25 \mathrm{mg} /$ day for a 5-day period. Complementary tests including tuberculosis, brucellosis, herpes simplex virus (HSV), and human immunodeficiency virus (HIV) were negative, and coronavirus PCR in CSF was also negative. The patient health condition gradually improved as well as her consciousness conditions that returned to normal level. The patient's fever stopped, and she was discharged from the hospital with a good general status.

As mentioned above, there are some reports on COVID19-induced encephalitis and encephalopathy. The best diagnosis of this patient was COVID-19 associated encephalopathy. COVID-19 disease can cause brain involvement in different ways. Firstly, coronavirus is a neurotropic virus, and through attaching to angiotensin converting enzyme (ACE) 2 receptor, which is located on cells surface such as astrocytes, can cause direct brain involvement. Moreover, another route of involvement is through olfactory nerve and bulb. Eventually, the hyper-inflammation induced by COVID-19 can cause acute disseminated encephalomyelitis (ADEM)-like involvement in patients with COVID-19 (Abdi et al. 2020). Whether the MS disease itself or the drugs consumed (such as immunosuppressive drugs) can increase the chance of developing brain involvement of COVID-19 is something that needs to be further explored. Nevertheless, one cannot overlook is the effect of cyclophosphamide as an immunosuppressive drug on developing COVID-19 associated encephalopathy in this patient.

Author contributions ANM contributed to conception and design, described the case study, analyzed data and prepared the manuscript.

\section{Compliance with ethical standards}

Conflict of interest The author declares that he has no conflict of interest.

\section{References}

Ahmad I, Rathore FA (2020) Neurological manifestations and complications of COVID-19: A literature review. J Clin Neurosci 77:8-12

Ye M, Ren Y, Lv T (2020) Encephalitis as a clinical manifestation of COVID-19. Brain Behav Immun 88:945-946

El-Zein RS, Cardinali S, Murphy C, Keeling T (2020) COVID-19-associated meningoencephalitis treated with intravenous immunoglobulin. BMJ Case Rep 13(9):e237364

Hayashi M, Sahashi Y, Baba Y, Okura H, Shimohata T (2020) COVID19-associated mild encephalitis/encephalopathy with a reversible splenial lesion. J Neurol Sci 415:116941

Berger JR (2020) COVID-19 and the nervous system. J Neurovirol 26(2):143-148

Abdi S, Ghorbani A, Fatehi F (2020) The association of SARS-CoV-2 infection and acute disseminated encephalomyelitis without prominent clinical pulmonary symptoms. J Neurol Sci 416:117001

Publisher's Note Springer Nature remains neutral with regard to jurisdictional claims in published maps and institutional affiliations. 